\title{
Detalhes, data e significado do quinto exemplar do mapa em gomos globulares de Waldseemüller recentemente descoberto na Biblioteca da Universidade de Munique
}

Detalles, fecha e importancia del quinto ejemplar del mapa en gajos globulares de Martin Waldseemüller recientemente descubierto en la Biblioteca de la Universidad de Múnich

Détails, date et importance du cinquième exemplaire de la carte en quartiers globulaires de Waldseemüller découvert récemment dans la Bibliothèque de l'Université de Munich

Details, Date, and Significance of the Fifth Set of Waldseemüller's Globe Gores Recently Discovered in the Munich University Library

\section{Chet Van Duzer}

Tradutor: David Palacios

\section{(2) OpenEdition}

Journals

\section{Edição electrónica}

URL: https://journals.openedition.org/terrabrasilis/483

DOI: 10.4000/terrabrasilis.483

ISSN: 2316-7793

\section{Editora}

Rede Brasileira de História da Geografia e Geografia Histórica

\section{Refêrencia eletrónica}

Chet Van Duzer, «Detalhes, data e significado do quinto exemplar do mapa em gomos globulares de Waldseemüller recentemente descoberto na Biblioteca da Universidade de Munique», Terra Brasilis [Online], 1 | 2012, posto online no dia 08 novembro 2012, consultado o 05 dezembro 2022. URL: http:// journals.openedition.org/terrabrasilis/483 ; DOI: https://doi.org/10.4000/terrabrasilis.483

Este documento foi criado de forma automática no dia 5 dezembro 2022.

All rights reserved 


\section{Detalhes, data e significado do quinto exemplar do mapa em gomos globulares de Waldseemüller recentemente descoberto na Biblioteca da Universidade de Munique}

Detalles, fecha e importancia del quinto ejemplar del mapa en gajos globulares de Martin Waldseemüller recientemente descubierto en la Biblioteca de la Universidad de Múnich

Détails, date et importance du cinquième exemplaire de la carte en quartiers globulaires de Waldseemüller découvert récemment dans la Bibliothèque de l'Université de Munich Details, Date, and Significance of the Fifth Set of Waldseemüller's Globe Gores Recently Discovered in the Munich University Library

Chet Van Duzer

Tradução : David Palacios

\section{NOTA DO EDITOR}

O presente artigo foi expressamente redigido pelo autor para o portal Razón Cartográfica - Red de História de las Geografías y Cartografías de Colombia [http://razoncartografica.com] e publicado em julho deste ano, um mês depois da descoberta do mapa na biblioteca da Universidade de Munique, como "Notícia Especial" [http://razoncartografica.com/ 2012/07/17/noticia-especial-detalles-fecha-e-importancia-del-quinto-ejemplar-del- 
mapa-en-gajos-del-globo-terraqueo-de-martin-waldseemuller-recientementedescubierto-en-munich/]. Tanto o autor quanto os administradores de Razão Cartográfica, casa irmã da RedeBrasilis, cederam amavelmente o artigo para ser publicado em versão brasileira neste número 1 da revista.

1 Junho passado, Gerlinde Geiselmann e Elke Humml, bibliotecárias da LudwigMaximilians-Universität (LMU) em Munique, descobriram um exemplar até agora desconhecido do mapa em gomos globulares (globe gores) desenhado originalmente pelo cartógrafo alemão Martin Waldseemüller (fig. 1).

Figura 1: 0 recentemente descoberto mapa em gomos de Martin Waldseemüller (posterior a 1507)

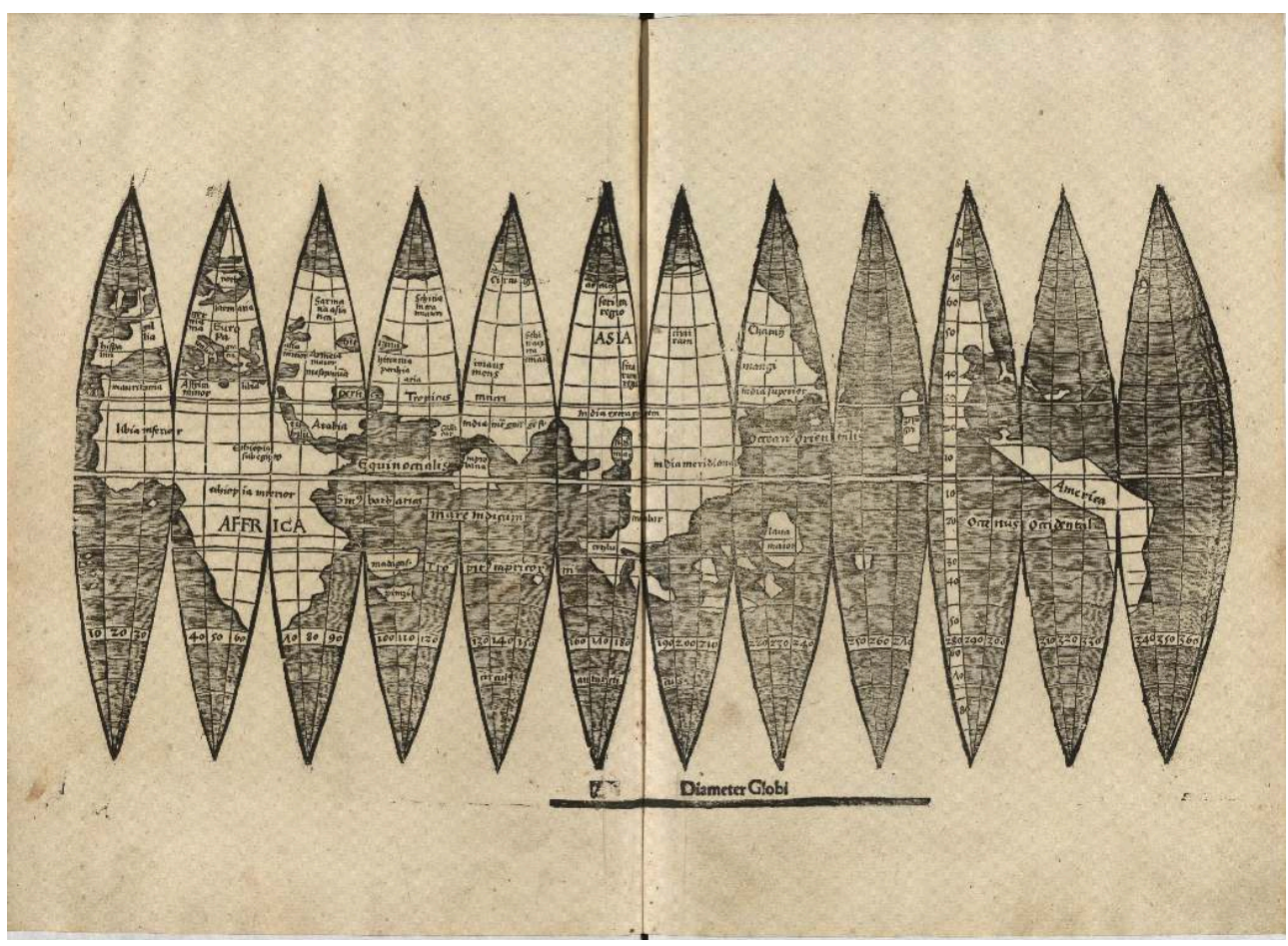

Cortesía de la Biblioteca da Ludwig-Maximilians-Universität de Munique (LMU, Cim. 107\#2). Imagem disponível em: [http://epub.ub.uni-muenchen.de/13138/].

O exemplar tinha sido encadernado no século XIX entre duas cópias da Perspectiva communis de Juan Peckham (uma publicada em 1504 e a outra por volta de 1510). ${ }^{1}$ Infelizmente, nada mais sabemos acerca da procedência deste exemplar do mapa em gomos. Até o momento conheciam-se unicamente outras quatro cópias destes gomos globulares, ${ }^{2}$ tratando-se portanto dum achado sensacional, tanto mais quanto este jogo de gomos é diferente dos outros. O propósito deste artigo é fornecer alguns detalhes acerca dos gomos e do significado da sua descoberta.

3 Martin Waldseemüller (c. 1475-1520), ${ }^{3}$ que passou a maior parte da sua vida na cidade de Saint-Dié, perto de Estrasburgo, é mais famoso por seu grande mapa do mundo de 1507, impresso em doze folhas e o primeiro em conferir o nome de América ao Novo Mundo (fig. 2 e 3). 
Figura 2: Mapa-múndi de Waldseemüller (1507)

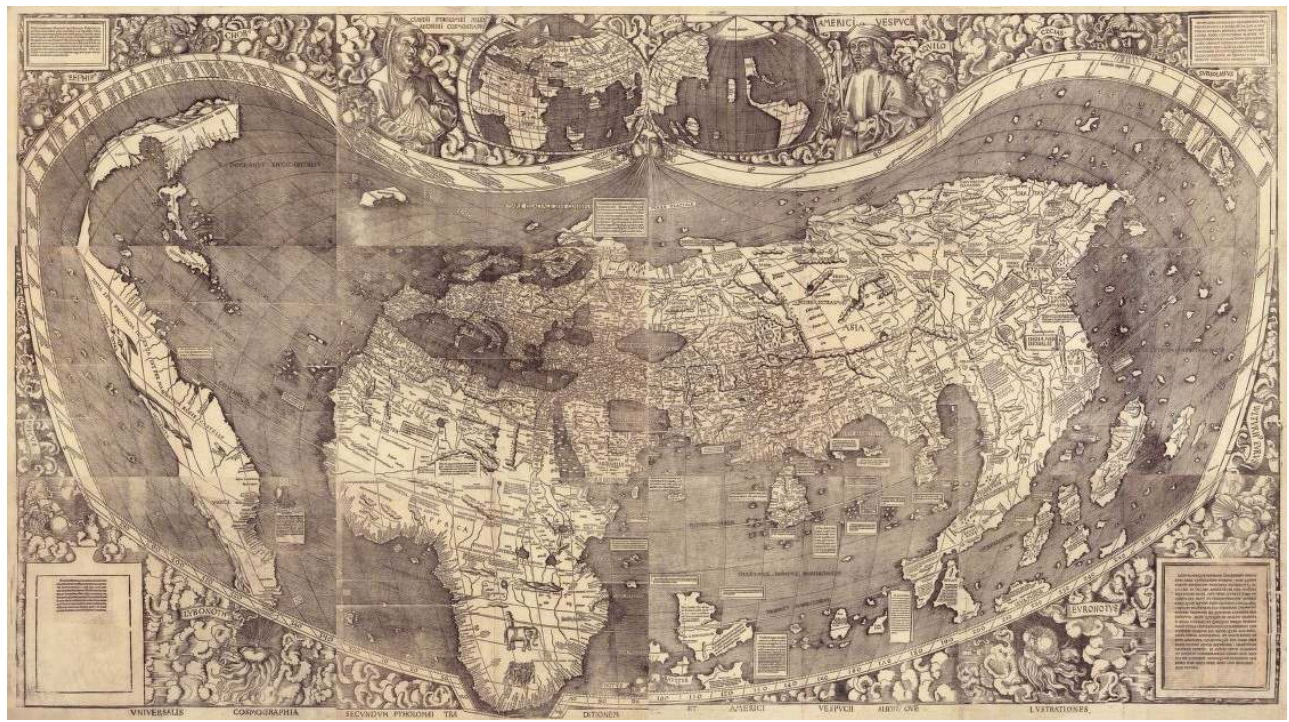

Cortesia da Biblioteca do Congresso [http://www.loc.gov/item/2003626426].

Figura 3: "América" no Mapa-múndi de Waldseemüller de 1507 (detalhe)

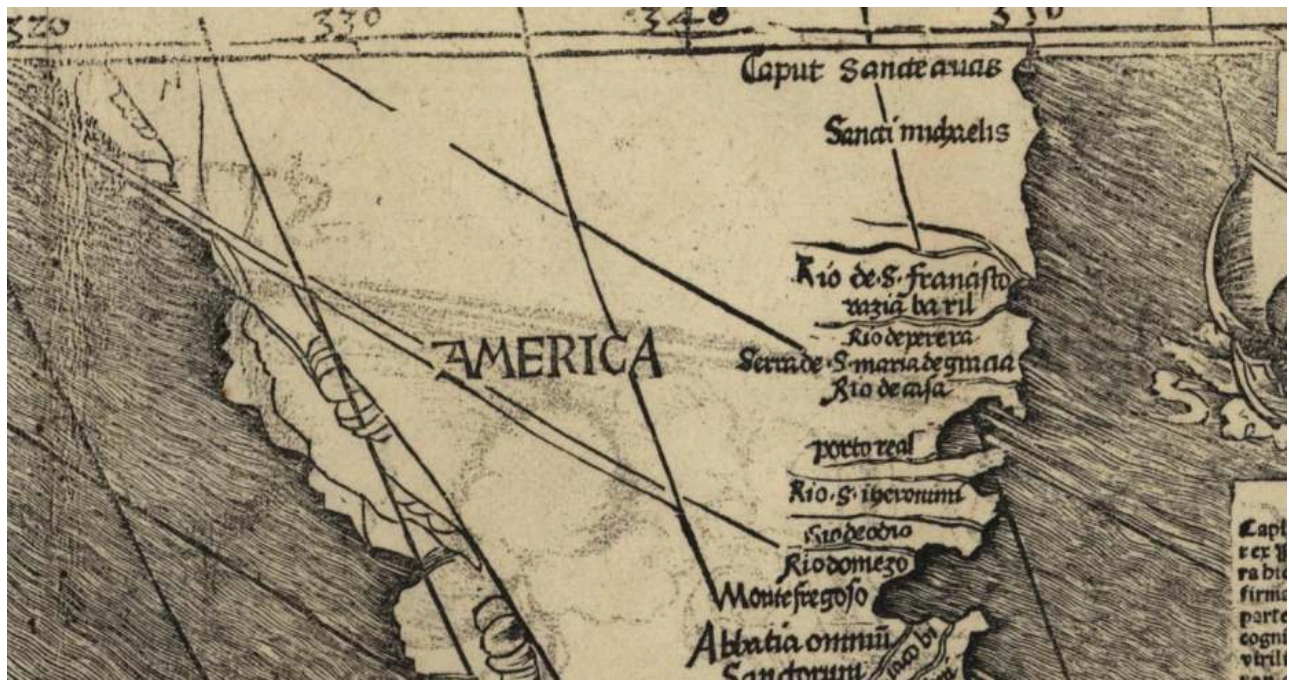

4 Este mapa sobrevive num único exemplar descoberto na Alemanha em 1901 e encontrase em exibição permanente na Biblioteca do Congresso em Washington DC. ${ }^{4}$ Este mapa vendia-se originalmente acompanhado de um jogo de gomos globulares (que dava também o nome de "América" ao Novo Mundo) e de um livro titulado Cosmographiae introductio (Introdução à Cosmografia), ${ }^{5}$ que continha uma explicação do nome dado às terras recentemente descobertas. Waldseemüller e seu coautor, Matthias Ringmann, apontaram que o explorador Amerigo Vespucci tinha recentemente descoberto uma "quarta parte" do mundo, e sugeriu que "uma vez que tanto a Europa quanto a Asia receberam seus nomes de mulheres, não vejo razão para alguém objetar com justiça chamar esta parte de Amerige, i.e., a terra de Amerigo, ou América, de Amérigo, seu descobridor, homem de grande habilidade". ${ }^{6}$ Esta denominação, efetuada na pequena cidade de Saint-Dié quinhentos anos atrás, continua a ter influência ainda hoje. 
Deste modo então os gomos globulares formavam parte do mesmo projeto cartográfico e cosmográfico que o famoso mapa do mundo de 1507, e participavam também na denominação das terras recém-descobertas. É tentador pensar que os gomos globulares eram vendidos junto com o mapa não só para ajudar o comprador a entender a projeção usada no mapa, mas também para enfatizar a ousadia de Waldseemüller de representar os $360^{\circ}$ da circunferência da terra numa época em que a maior parte da superfície do planeta era ainda desconhecida.

6 Nas matérias sobre a descoberta do quinto jogo de gomos, Sven Kuttner, Bibliotecário de Livros Raros na Universidade de Munique, notou algumas diferencias entre o novo exemplar e os quatro outros. ${ }^{7}$ Nos quatro mapas previamente conhecidos, a maioria dos segmentos dos gomos possuem uma borda preta grossa (fig. 4), entanto que o jogo da LMU não.

Figura 4. Exemplar da Bayerische Staatsbibliothek [1507]

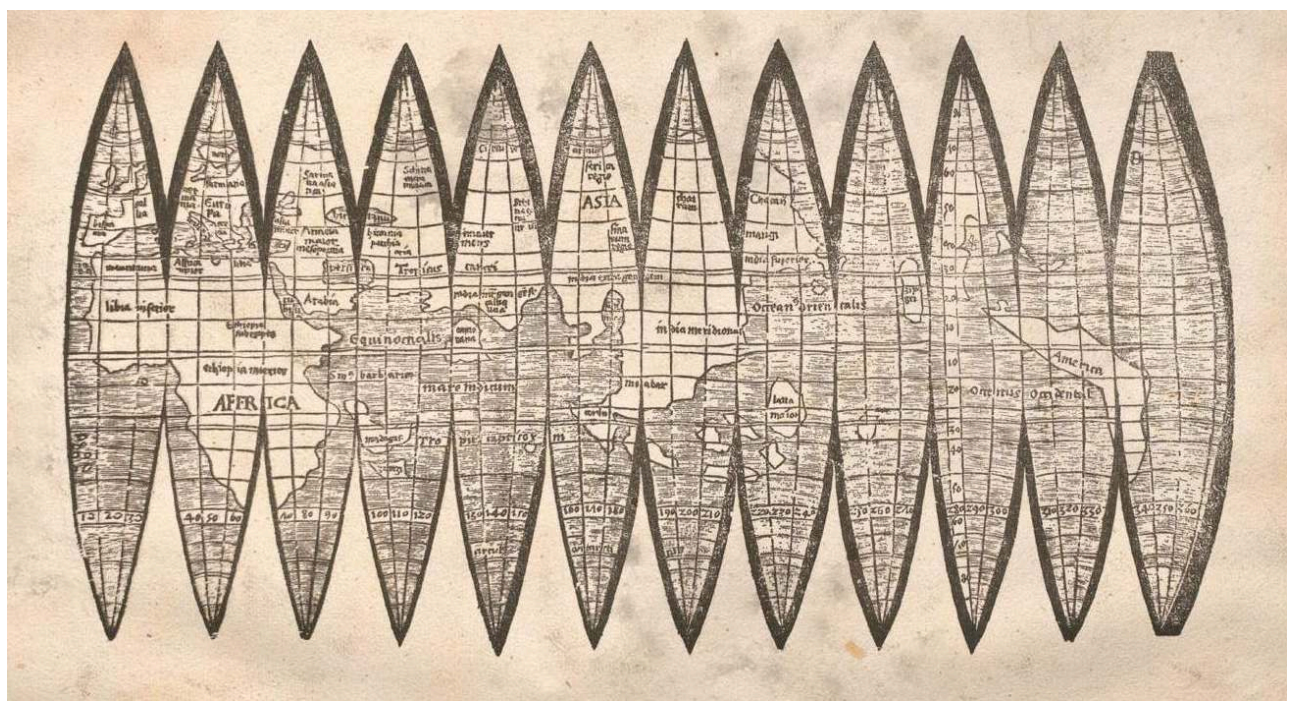

Disponível em: [http://daten.digitale-sammlungen.de/bsb00013398/image_1]

Nos quatro exemplares até agora conhecidos, Calecute encontra-se no quinto segmento dos gomos, na costa Malabar ao nordeste da ilha de Taprobana, entanto que no exemplar da LMU encontra-se no quarto segmento, ao noroeste da ilha. Kuttner aponta também que o estilo da hachura que representa as águas dos oceanos e os mares é diferente, que as formas de algumas das letras é diferente, e também que a marcad'água coincide com a de um livro de $1518,{ }^{8}$ o que sugere que os gomos foram impressos depois da primeira edição de 1507.

Debe especificar-se claramente que o exemplar da LMU foi impresso a partir de uma prancha completamente diferente do da edição de 1507, havendo outras diferencias importantes de detalhe entre o jogo da LMU e os quatro outros conhecidos que confirmam que representa uma edição diferente, anteriormente desconhecida. 0 mais surpreendente é que o exemplar da LMU inclui na parte de baixo uma linha grossa com a indicação "Diameter Globi" justo encima, indicando o diâmetro da esfera sobre a qual os gomos podiam ser colados. Estas palavras foram impressas usando tipos fixados na prancha dos gomos, uma técnica usada nos mapas de Waldseemüller de 1507 e de $1516 .{ }^{9}$ O tipo de letra é o mesmo usado por Waldseemüller na sua Cosmographiae introductio assim como em alguns dos blocos de texto que aparecem em seus mapas de 1507 e 1516, 
${ }^{10}$ no entanto esta parte do bloco foi sobre-entintada quando o exemplar da LMU foi impresso (as letras estão pesadamente impressas e a tinta sangrou no papel ao redor de cada letra). A adição das palavras "Diameter Globi" parece dirigida a orientar o comprador dos gomos, pois sem esta indicação ele teria de medir a longitude equatorial do mapa e dividir esta distância entre $\pi$ com o fim de determinar o diâmetro correto. Por sinal, esta frase é uma boa indicação de que embora o título da Cosmographiae introductio afirmasse que era para ela ser acompanhada duma Universalis Cosmographiae descriptio tam in solido quam plano, "um mapa do mundo tanto em duas quanto em três dimensões", os gomos eram vendidos como gomos e não já colados a uma esfera.

Em geral os gomos da LMU seguem a cartografia dos outros gomos muito de perto, com variações muito mínimas nos contornos das costas que parecem acidentais. Há duas mudanças que parecem ser intencionais. Em primeiro lugar, nos mapas em gomos até agora conhecidos, a forma do oeste da Cuba não está bem definida, entanto que no mapa da LMU encontra-se mais articulada -mais próxima da imagem da ilha no mapa grande de 1507. Assim, o criador da prancha para esta versão dos gomos tentou fazê-los um tanto mais acurados neste respeito do que na outra versão.

A segunda mudança aparentemente intencional na cartografia dos gomos é muito mais interessante: envolve a forma de Madagascar. É claro que originalmente na prancha do mapa da LMU a ilha tinha a caraterística forma de "L" que Waldseemüller deu-lhe em seu mapa grande de 1507 assim como nos outros quatro exemplares existentes do seu globos em gomos. Waldseemüller deu esta forma à ilha por influência de Henricus Martellus, pois a ilha tem esta forma num mapa evidentemente desenhado por Martellus que foi impresso por Francesco Rosselli. ${ }^{11}$ Nos gomos da LMU, a prancha foi modificada com o fim de que a parte da ilha ao norte do Trópico de Capricórnio fosse eliminada e substituída por hachuras indicando água, embora o antigo contorno da ilha seja ainda visível. Embora Waldseemüller mostre Madagascar estendendo-se ao norte do Trópico de Capricórnio no novo mapa do mundo da sua edição de 1513 da Geographia de Ptolomeu (o chamado "Mapa do Almirante", fig. 5), ${ }^{12}$ no seu novo mapa do sul da África (fig. 6), a ilha encontra-se completamente ao sul do Trópico. 
Figura 5: 0 chamado “Mapa do Almirante" na edição de 1513 da Geografia de Ptolomeu

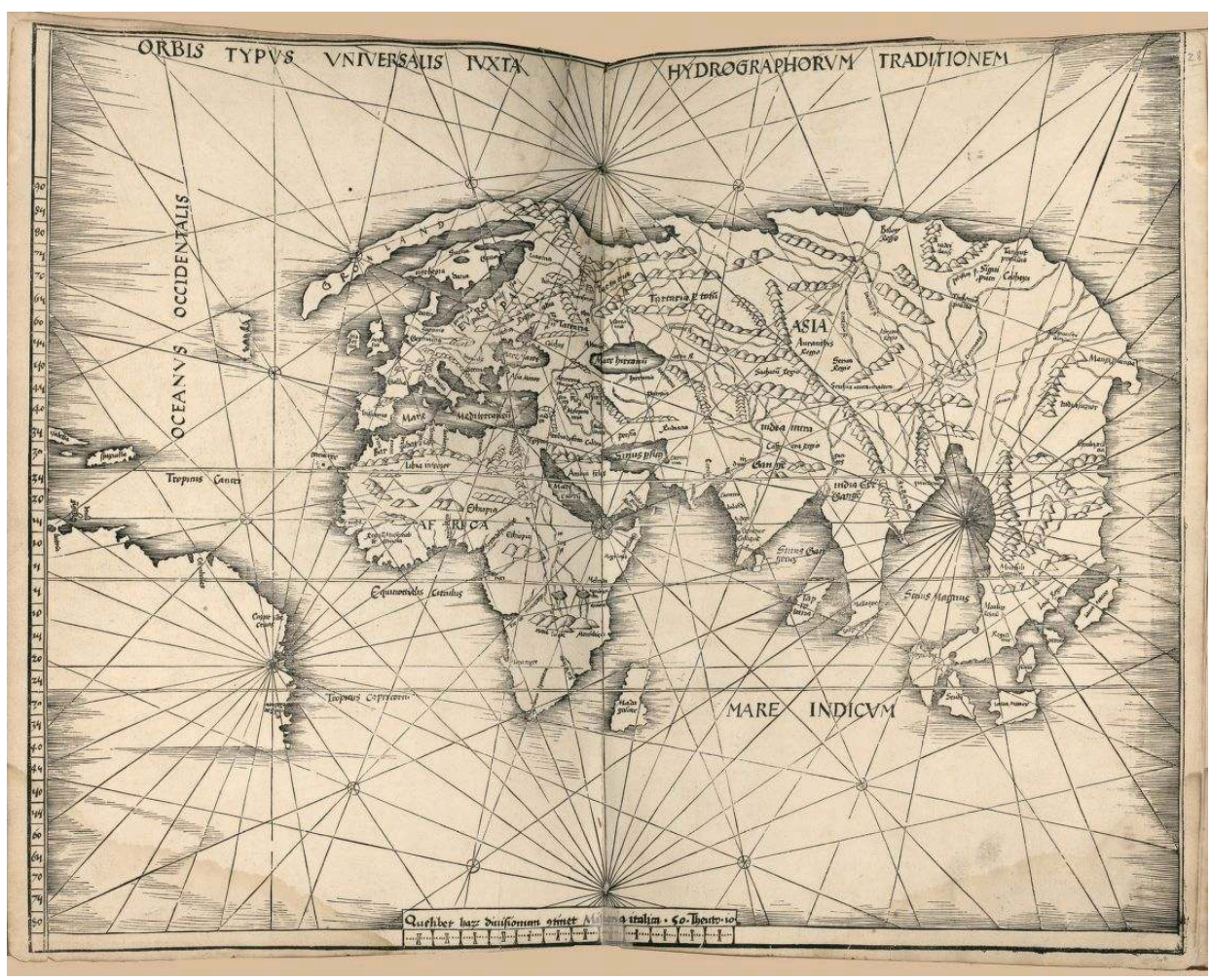

Cortesia da Biblioteca do Congresso [http://memory.loc.gov/cgi-bin/map_item.pl?data=/home/www/ data/gmd//gmd3m/g3200m/g3200m/gct00262/ca000131.jp2\&style=gmd\&itemLink=r?ammem/ gmd:@field\%28NUMBER+@band\%28g3200m+gct00262\%29\%29\&title=Claudii\%20Ptolemei\%20viri\%20Alexandrini\%20mathematice\%20disc \%20ceteris\%20ante\%20lucubratorum\%20multo\%20prestantius\%20...+-+Map\%20of\%20the\%20world]. 
Figura 6: 0 novo mapa do sul da África na edição de 1513 da Geografia Ptolomeu

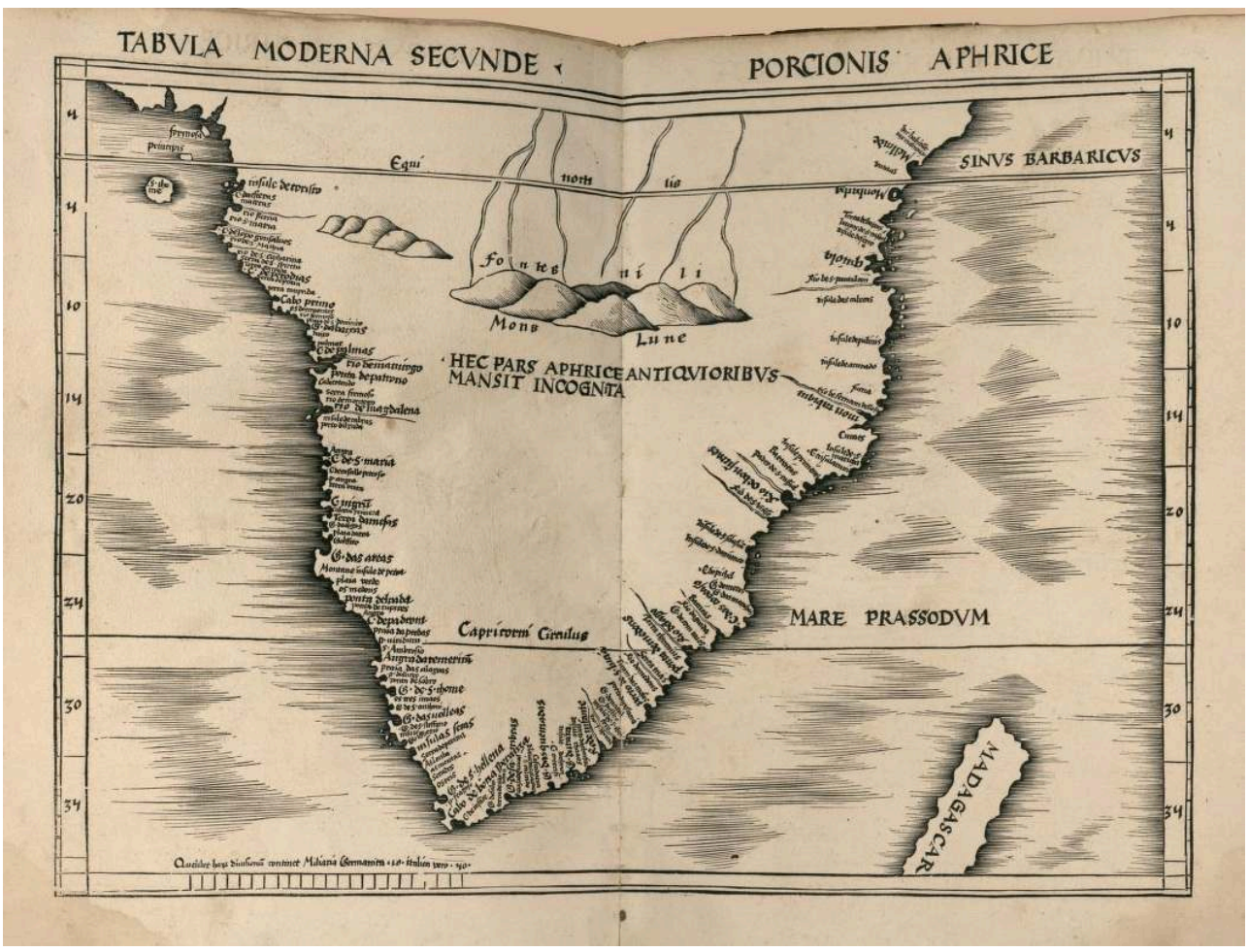

Cortesia da Biblioteca do Congresso [http://memory.loc.gov/cgi-bin/map_item.pl?data=/home/www/ data/gmd//gmd3m/g3200m/g3200m/gct00262/ca000155.jp2\&style=gmd\&itemLink=r?ammem/ gmd:@field\%28NUMBER+@band\%28g3200m+gct00262\%29\%29\&title=Claudii\%20Ptolemei\%20viri\%20Alexandrini\%20mathematice\%20disci \%20ceteris\%20ante\%20lucubratorum\%20multo\%20prestantius\%20...+-+Map\%20of\%20Africa].

Parece ser então que a modificação de Madagascar nos gomos da LMU baseou-se no seguimento por parte de Waldseemüller duma nova fonte com respeito à posição da ilha: a carta náutica de Nicolo de Caverio de c. 1504 (fig. 7), ${ }^{13}$ que também localiza a ilha no sul, ao contrário do mapa de Martellus que era dez ou quinze anos mais velho. 
Figura 7: Sul da África e Madagascar na carta náutica de Nicolo de Caverio (c. 1504)

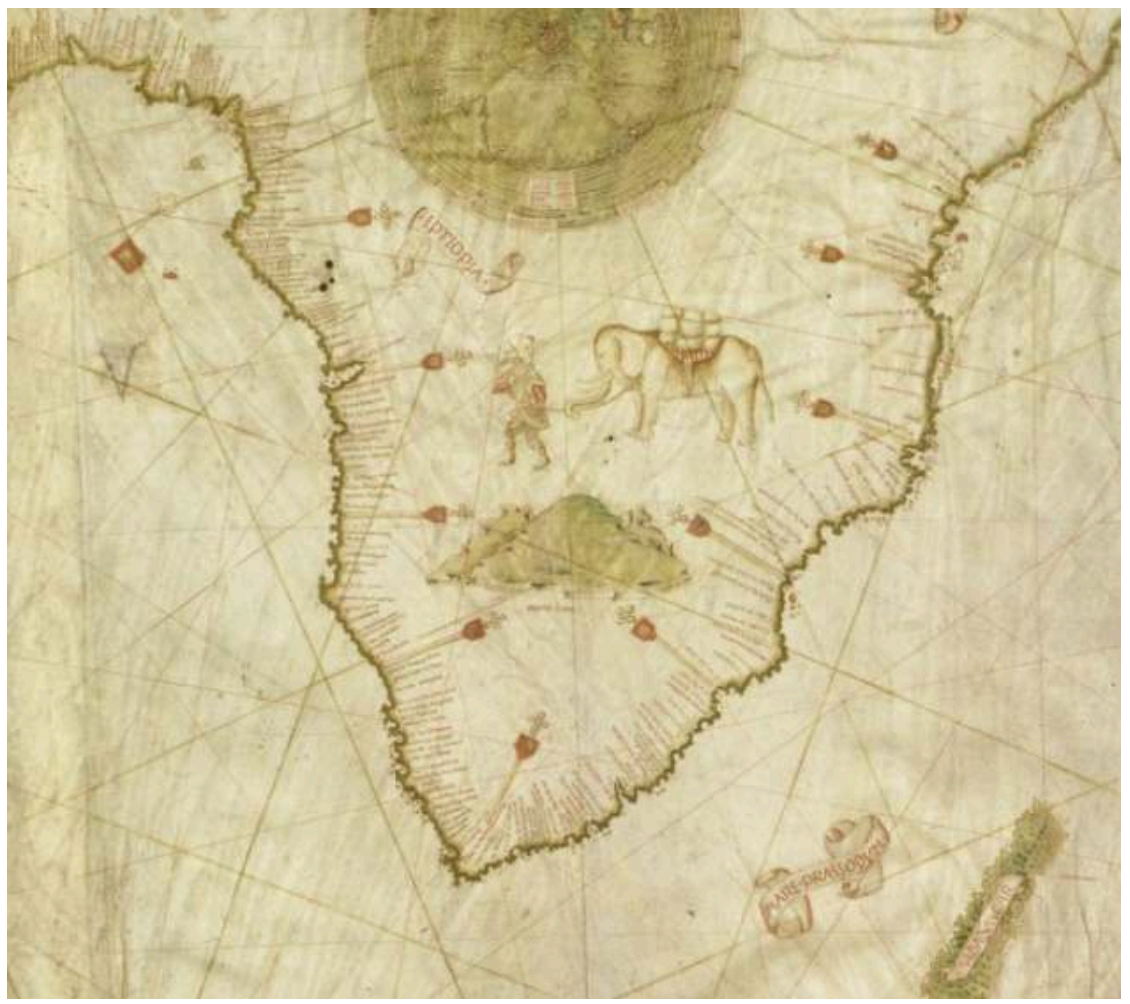

Disponível na Gallica (Biblioteca Nacional da França) [http://gallica.bnf.fr/ark:/12148/btv1b7759102x].

Desta forma a mudança em Madagascar nos gomos da LMU confirma a evidência da marca-d'água de que o mapa é posterior a 1507. Embora Waldseemüller tivesse a carta de Caverio quando fez seu mapa de 1507, e de que a seguisse de perto na toponímia do Novo Mundo e da África, ${ }^{14}$ foi só em data posterior que o seguiu com respeito à posição de Madagascar.

É mais difícil saber como interpretar a mudança na posição de Calicut aludida acima. A geografia do novo mapa do mundo de Waldseemüller, na edição de 1513 da Geografia de Ptolomeu, é tão diferente da do mapa de 1507 que é difícil comparar detalhes desta natureza, tanto mais quando o mapa de 1513 não indica a longitude. Mas há evidência interessante que sugere que a posição de Calecute nos gomos da LMU representa uma atualização com respeito aos outros gomos sobreviventes e ao mapa de 1507. No seu globo terrestre de 1515, Johann Schöner seguiu o mapa de Waldseemüller de 1507 muito de perto, ${ }^{15}$ mas coloca Calecute ao noroeste de Taprobana, numa posição mais parecida com a dos gomos da LMU.

O mapa em gomos da LMU contém uma outra importante caraterística que oferece orientação quanto à sua data: inclui o nome "América" no Novo Mundo. Este nome tinha sido proposto na Cosmographiae introductio e incluído no grande mapa de 1507 assim como nos outros gomos globulares sobreviventes, mas não é usado na edição de Waldseemüller de 1513 da Geografia de Ptolomeu, nem no novo mapa do mundo (o Mapa do Almirante) nem no mapa do Novo Mundo, o Atlântico, Europa Ocidental e África Ocidental que lhe segue imediatamente (fig. 8). O nome também não aparece na sua Carta marina de 1516: Waldseemüller tinha chegado à conclusão de que Cristóvão Colombo e não Amerigo Vespucci era o descobridor do Novo Mundo. Assim, enquanto a prancha do mapa da LMU foi com certeza elaborada após 1507, e depois de 
Waldseemüller abandonar a concepção de Martellus com respeito à localização de Madagascar, ela foi feita provavelmente antes de 1513, e com total certeza antes de 1516.

Figura 8: 0 mapa do Atlântico e seus arredores na edição de 1513 da Geografia Ptolomeu

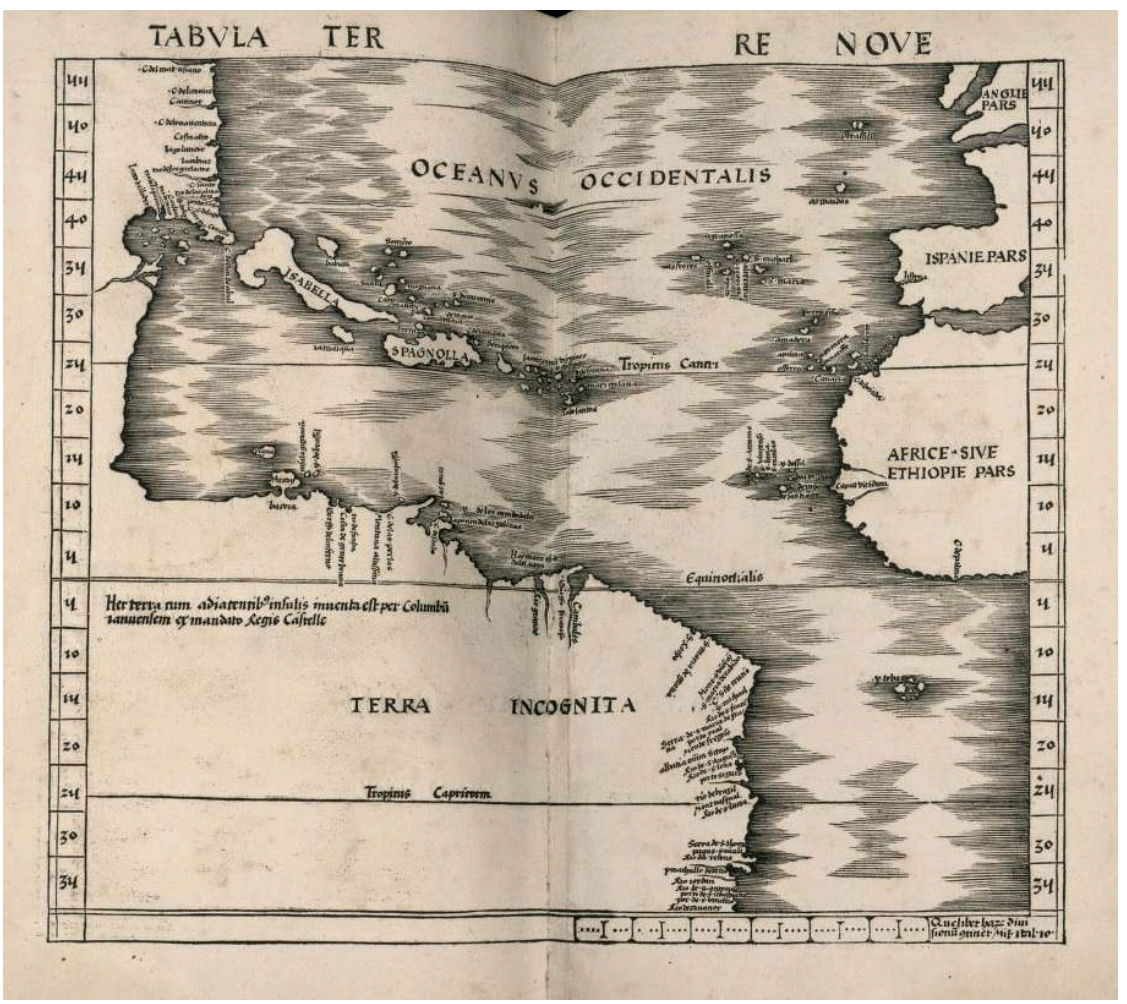

Cortesia da Biblioteca do Congresso [http://memory.loc.gov/cgi-bin/map_item.pl?data=/home/www/ data/gmd//gmd3m/g3200m/g3200m/gct00262/ca000133.jp2\&style=gmd\&itemLink=r?ammem/ gmd:@field\%28NUMBER+@band\%28g3200m+gct00262\%29\%29\&title=Claudii\%20Ptolemei\%20viri\%20Alexandrini\%20mathematice\%20disc \%20ceteris\%20ante\%20lucubratorum\%20multo\%20prestantius\%20...+-+Map].

15 Há um outro mapa adscrito à Waldseemüller que, de uma forma muito interessante, perece encaixar neste mesmo período de transição entre o grande mapa do mundo de 1507 e a edição de 1513 de Ptolomeu: trata-se do chamado "Mapa Stevens-Brown" da Biblioteca John Carter Brown (fig. 9) ${ }^{16}$ Este mapa não datado é muito semelhante de fato ao novo mapa do mundo de 1513 de Ptolomeu (o Mapa do Almirante), no entanto foi impresso a partir duma prancha diferente, e inclui o nome de "América", o que a versão do mapa no Ptolomeu de 1513 não faz. Assim, os gomos da LMU constituem uma nova versão dos gomos de 1507, com a modificação de Madagascar que coloca esta parte do mapa mais em linha com o pensamento posterior de Waldseemüller, enquanto o mapa Stevens-Brown é muito semelhante ao mapa do mundo do seguinte grande projeto cartográfico de Waldseemüller, a edição de 1513 de Ptolomeu, mas retem um elemento (o nome "América") que remonta a seus trabalhos anteriores. 
Figura 9: 0 mapa Stevens-Brown [1513]

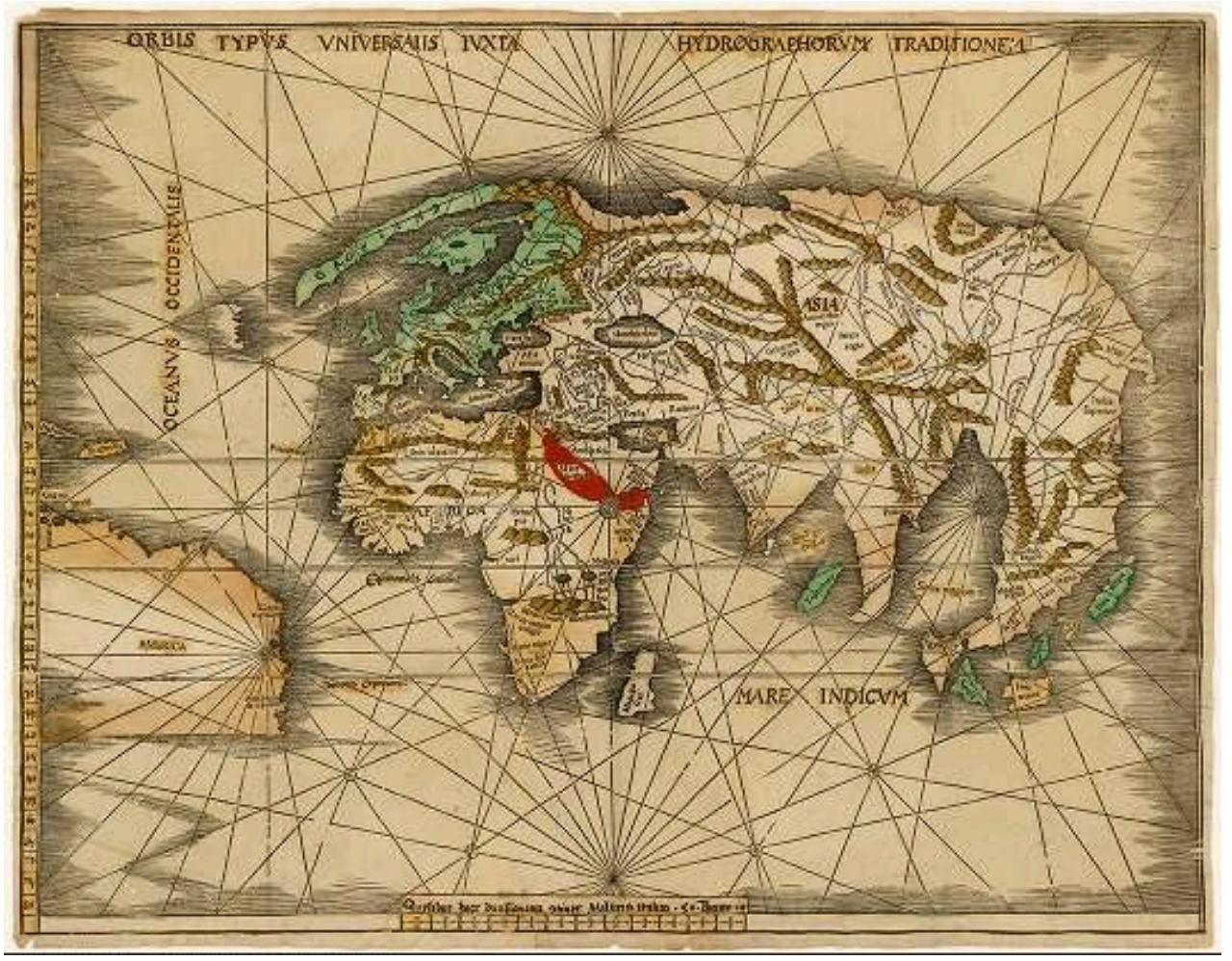

Cortesia da Biblioteca John Carter Brown da Brown University [http://jcb.lunaimaging.com/luna/ servlet/s/9nz9qy]

Waldseemüller e seus colegas em Saint-Dié e Estrasburgo eram experimentadores, sempre procurando a melhor forma de fazer as coisas. Para mencionar só dois exemplos, em algumas copias da edição de Ptolomeu de 1513 no mapa das Ilhas Britânicas o mar está pintado num tom verde-cinza, e, em todas as cópias do livro, o mapa da Lorena está impresso em três cores (fig. 10), sendo ambas experiências primitivas de impressão colorida de mapas. ${ }^{17}$ Os gomos da LMU fornecem evidencia adicional do seu desejo de melhorar o que tinham feito antes. Adicionalmente, a existência de uma edição separada dos gomos pode talvez outorgar crédito a uma afirmação de Waldseemüller num bloco de texto da sua Carta marina de 1516 segundo a qual ele tinha impresso 1000 copias do seu anterior mapa (i. e. o mapa de 1507). Talvez o aspecto mais importante do mapa em gomos da LMU é que verte luz sobre as atividades do círculo de Waldseemüller em Saint-Dié entre a produção de seu grande mapa de 1507 e o Ptolomeu de 1513, período do qual em tudo caso sabemos muito pouco. 
Figura 10: 0 mapa da Lorena impresso em cores na edição de 1513 da Geografia Ptolomeu

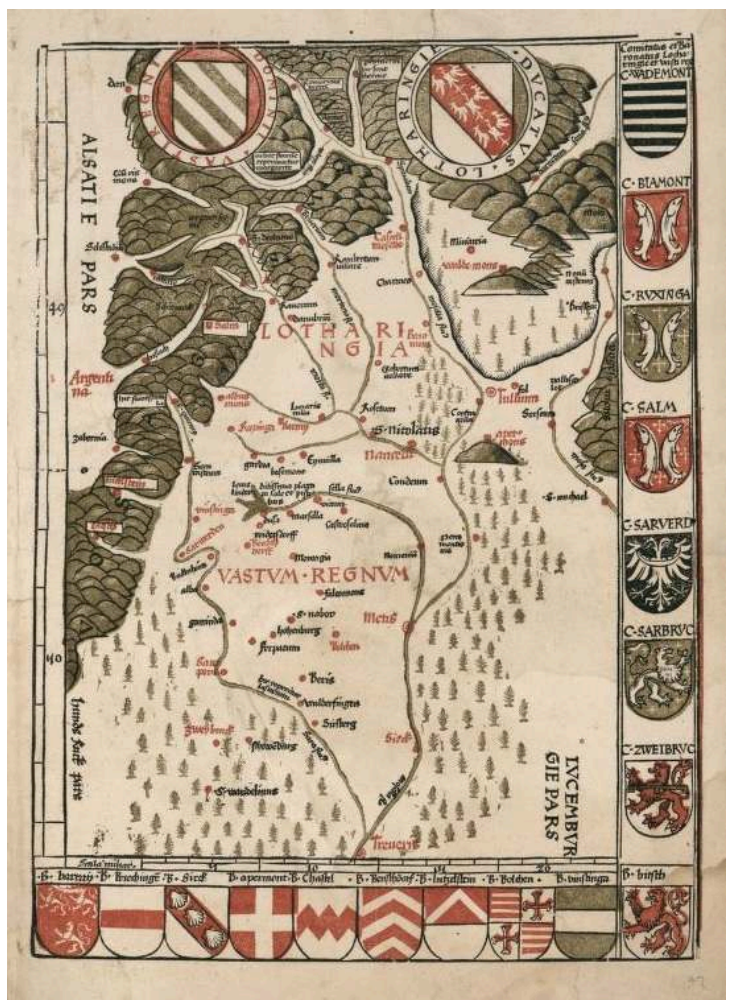

Cortesia da Biblioteca do Congresso [http://memory.loc.gov/cgi-bin/map_item.pl?data=/home/www/ data/gmd//gmd3m/g3200m/g3200m/gct00262/ca000168.jp2\&style=gmd\&itemLink=r?ammem/ gmd:@field\%28NUMBER+@band\%28g3200m+gct00262\%29\%29\&title=Claudii\%20Ptolemei\%20viri\%20Alexandrini\%20mathematice\%20disci \%20ceteris\%20ante\%20lucubratorum\%20multo\%20prestantius\%20...+-+Map].

\section{BIBLIOGRAFIA}

ALMAGIÀ, Roberto, “On the Cartographic Work of Francesco Rosselli,” Imago Mundi 8, 1951, p. $27-34$

Americana vetustissima: Fifty Books, Manuscripts, \& Maps Relating to America from the First Fifty Years after its Discovery (1493-1542), New York: H. P. Kraus, 1990

CAVALLO, Guglielmo, ed., Cristoforo Colombo e l'apertura degli spazi: Mostra storico-cartografica, Rome: Istituto Poligrafico e Zecca dello Stato, 1992

CAMPBELL, Tony, The Earliest Printed Maps, 1472-1500, London: British Library, 1987

Cartography, Including the Waldseemüller Gores : Wednesday 8 June 2005. London: Christie, Manson \& Woods Ltd., 2005

D'AVEZAC, M., Martin Hylacomylus Waltzemüller, ses ouvrages et ses collaborateurs, Paris: Challamel âiné, 1867 
DUZER, Chet Van, Johann Schöner's Globe of 1515: Transcription and Study, Philadelphia: American Philosophical Society, $2010=$ Transactions of American Philosophical Society 100.5

DUZER, Chet Van \& LARGER, Benoit, “Martin Waldseemüller's Death Date,” Imago Mundi 63.2, 2011, pp. 217-219.

Erster gedruckter Globus, Martin Hylacomylus (Waltzemüller): gehört wahrscheinlich zu seinem 1509 herausgegeben Buch Globus mundi'; Zweiter gedruckter Globus, Peter Apianus: in dessen 'Cosmographicus liber', 1524, sich bereits ein Globus mit Gestell abgebildet befinden, Vienna, 1879

FISCHER, Joseph \& WIESER, Franz Ritter von, Die älteste Karte mit dem Namen Amerika aus dem Jahre 1507 und die Carta marina aus dem Jahre 1516 des M. Waldseemüller (Ilacomilus), Innsbruck: Wagner'schen Universitäts-Buchhandlung, 1903

, The Cosmographiae introductio of Martin Waldseemüller in Facsimile, Followed by the Four Voyages of Amerigo Vespucci, with their Translation into English, New York: The United States Catholic Historical Society, 1907; Amsterdam: Theatrum orbis terrarum, 1968

GÉRARDIN, Jean-Marie, "1508-2008: A propos de la première carte imprimée du duché de Lorraine et du Vastum Regnum," L'Annuaire de la Société du Val de Villé 33, 2008, pp. 57-77

HARRIS, Elizabeth, “The Waldseemüller World Map: A Typographic Appraisal,” Imago Mundi 37, 1985, pp. 30-53

HARRISSE, Henry, Bibliotheca americana vetustissima: A Description of Works Relating to America, Published between the Years 1492 and 1551, New York: G. P. Philes, 1866 The Discovery of North America, London: H. Stevens, 1892; Amsterdam: N. Israel, 1961

HESSLER, John W., The Naming of America: Martin Waldseemüller's 1507 World Map and the 'Cosmographiae introductio', London: D. Giles, 2008

HESSLER, John W. \& DUZER, Chet Van, Seeing the World Anew: The Radical Vision of Martin Waldseemüller's 1507 \& 1516 World Maps, Washington, DC: Library of Congress and Delray Beach, FL: Levenger Press, 2012.

JOHNSON, Christine R., "Renaissance German Cosmographers and the Naming of America," Past \& Present 191 (2006), pp. 3-43

KARROW, Robert W. Jr., Mapmakers of the Sixteenth Century and their Maps, Chicago: The Newberry Library - Speculum Orbis Press, 1993

LAUBENBERGER, Franz (trans. Steven Rowan), "The Naming of America," The Sixteenth Century Journal 13.4, 1982, pp. 91-113

LESTER, Toby, The Fourth Part of the World: The Race to the Ends of the Earth, and the Epic Story of the Map That Gave America its Name, New York: Free Press, 2009

LINDBERG, David C., John Pecham and the Science of Optics: Perspectiva Communis, Madison:University of Wisconsin Press, 1970

McCOSKRY, Samuel Stanton, “The Admiral's Map: What Was It? And Who the Admiral?” Isis 22.2, 1935, pp. 511-515

NEBENZAHL, Kenneth, Atlas of Columbus and the Great Discoveries, Chicago: Rand McNally, 1990

NEWTON, Henry Stevens, The First Delineation of the New World and the First Use of the Name America on a Printed Map, London: H. Stevens, Son and Stiles, 1928 
PICCARD, Gerhard, Die Wasserzeichenkartei Piccard im Hauptstaatsarchiv Stuttgart: Findbuch, Stuttgart: Kohlhammer, 1961-1997

SHIRLEY, Rodney W., The Mapping of the World: Early Printed World Maps, 1472-1700, London: Holland Press, 1983

"Karte der Britischen Inseln von 1513: eine der ersten farbig gedruckten Karten," Cartographica Helvetica 19-20, 1999, pp. 13-17

STEVENSON, Edward L., Marine World Chart of Nicolo de Caneiro Januensis 1502 (circa), New York: American Geographical Society and the Hispanic Society of America, 1908

Terrestrial and Celestial Globes: Their History and Construction, New Haven: Yale University Press, 1921

WOLFF, Hans, "Martin Waldseemüller: The Most Important Cosmographer in a Period of Dramatic Scientific Change”, in WOLFF, Han ( ed.), America: Early Maps of the New World, trans. Hugh Beyer et al., Munich: Prestel, 1992

\section{NOTAS}

1. Este trabalho foi traduzido por David C. Lindberg como John Pecham and the Science of Optics: Perspectiva Communis (Madison:University of Wisconsin Press, 1970).

2. Os quatro outros exemplares do mapa em gomos encontram-se em poder da Universidade de Minnesota [https://www.lib.umn.edu/apps/bell/WaldsWebsite/M\&MwaldsHOME.html], na Bayerische Staatsbibliothek [http://daten.digitale-sammlungen.de/bsb00013398/image_1], no Stadtbücherei Offenburg [http://www.neueweltundalteswissen.de/] e na Charles Frodsham and Co. Ltd., adquirido no leilão de Christie's de junho 8 de 2005 [http://www.christies.com/ lotfinder/lot_details.aspx?intObjectID=4516015]. Os gomos são discutidos em: Henry Harrisse, The Discovery of North America (London: H. Stevens, 1892; Amsterdam: N. Israel, 1961), pp. 440-442, no. 67, e 467-468, no. 82; Joseph Fischer and Franz von Wieser in The Cosmographiae introductio of Martin Waldseemüller in Facsimile, Followed by the Four Voyages of Amerigo Vespucci, with their Translation into English (New York: The United States Catholic Historical Society, 1907), pp. 23-30; Edward L. Stevenson, Terrestrial and Celestial Globes: Their History and Construction (New Haven: Yale University Press, 1921), vol. 1, pp. 70-71; Americana vetustissima: Fifty Books, Manuscripts, \& Maps Relating to America from the First Fifty Years after its Discovery (1493-1542) (New York: H. P. Kraus, 1990), pp. 30-31; e Cartography, Including the Waldseemüller Gores: Wednesday 8 June 2005 (London: Christie, Manson \& Woods Ltd., 2005). Um fac-similar dos gomos foi publicado como Erster gedruckter Globus, Martin Hylacomylus (Waltzemüller): gehört wahrscheinlich zu seinem 1509 herausgegeben Buch Globus mundi'; Zweiter gedruckter Globus, Peter Apianus: in dessen 'Cosmographicus liber', 1524, sich bereits ein Globus mit Gestell abgebildet befinden (Vienna, 1879). Um fac-similar globular foi também publicado por Greaves \& Thomas de Londres em 2007.

3. Para informação biográfica de Waldseemüller ver Hans Wolff, "Martin Waldseemüller: The Most Important Cosmographer in a Period of Dramatic Scientific Change," in Hans Wolff, ed., America: Early Maps of the New World, trans. Hugh Beyer et al. (Munich: Prestel, 1992), pp. 111-126; and Chet Van Duzer and Benoît Larger, "Martin Waldseemüller's Death Date," Imago Mundi 63.2 (2011), pp. 217-219 [http://www.jstor.org/stable/23016854].

4. Para o trabalho cartográfico de Waldseemüller em geral ver Robert W. Karrow, Jr., Mapmakers of the Sixteenth Century and their Maps (Chicago: The Newberry Library - Speculum Orbis Press, 1993), pp. 568-583. O mapa do mundo de Waldseemüller de 1507 tem sido publicado em facsimilar em Joseph Fischer and Franz Ritter von Wieser, Die älteste Karte mit dem Namen Amerika aus 
dem Jahre 1507 und die Carta marina aus dem Jahre 1516 des M. Waldseemüller (Ilacomilus) (Innsbruck: Wagner'schen Universitäts-Buchhandlung, 1903; Amsterdam: Theatrum orbis terrarum, 1968), com uma boa mas breve introdução; e agora em John W. Hessler e Chet Van Duzer, Seeing the World Anew: The Radical Vision of Martin Waldseemüller's 1507 \& 1516 World Maps (Washington, DC: Library of Congress and Delray Beach, FL: Levenger Press, 2012). Há duas excelentes imagens do mapa em alta resolução no site da Biblioteca do Congresso [http://www.loc.gov/item/2003626426 e http://memory.loc.gov/cgi-bin/map_item.pl?data=/home/www/data/gmd/gmd3/g3200/ g3200/ct000725C.jp2\&style=gmd\&itemLink=D?gmd:1:./temp/

ammem_ZgFX::\&title=Universalis\%20cosmographia\%20secundum\%20Ptholomaei\%20traditionem\%20et\%20Americi\%20Vespucii\%20ali 5. Sobre a Cosmographiae introductio ver Henry Harrisse, Bibliotheca americana vetustissima: A Description of Works Relating to America, Published between the Years 1492 and 1551 (New York: G. P. Philes, 1866), \#44-47, pp. 89-96 e M. d'Avezac, Martin Hylacomylus Waltzemüller, ses ouvrages et ses collaborateurs (Paris: Challamel âiné, 1867), pp. 36-59. A primeira edição foi reproduzida em facsímile e traduzida ao inglês por Fischer e von Wieser em The 'Cosmographiae introductio' of Martin Waldseemüller. Uma nova tradução da Cosmographiae introductio feita por John Hessler, junto com uma análise detalhada da obra, pode encontrar-se em seu livro The Naming of America: Martin Waldseemüller's 1507 World Map and the 'Cosmographiae introductio' (London: D. Giles, 2008).

6. Ver Fischer e von Wieser, The 'Cosmographiae introductio' of Martin Waldseemüller, chapter 9, p. xxx na tradução latina, e p. 70 na inglesa; e Hessler, The Naming of America, p. 101. O fac-símile e tradução de Fisher e von Wieser está disponível em www.archive.org [http://archive.org/details/ cosmographiaeint00walduoft]; a primeira edição da obra (abril de 1507) está disponível em PDF de alta qualidade via o catálogo on-line da Biblioteca do Congresso [http://lccn.loc.gov/ 05016176], e um PDF de alta qualidade da edição de agosto de 1507 está disponível no site da LMU [http://epub.ub.uni-muenchen.de/11046/1/Cim._74.pdf]. Para uma discussão da nominação de América ver Franz Laubenberger (trans. Steven Rowan), "The Naming of America," The Sixteenth Century Journal 13.4 (1982), pp. 91-113 [http://www.jstor.org/stable/2540012], com algumas correções em Christine R. Johnson, "Renaissance German Cosmographers and the Naming of America," Past \& Present 191 (2006), pp. 3-43 [http://www.jstor.org/stable/4125188].

7. Notícias da descoberta começaram a ser amplamente disseminadas em julho 3 de 2012 . Ver por exemplo o Chronicle of Higher Education de julho 7 de 2012 em [http://chronicle.com/blogs/tweed/ unknown-copy-of-map-that-gave-america-its-name-turns-up-in-munich-library/29773].

8. Gerhard Piccard, Die Wasserzeichenkartei Piccard im Hauptstaatsarchiv Stuttgart: Findbuch (Stuttgart: Kohlhammer, 1961-1997), vol. 4.2, p. 180.

9. Ver Elizabeth Harris, "The Waldseemüller World Map: A Typographic Appraisal," Imago Mundi 37 (1985), pp. 30-53 [http://www.jstor.org/stable/1150824].

10. Esta é a fonte que Harris designa como R1. Ver op. cit. pp. 42-45.

11. O mapa impresso por Rossellli encontra-se em Florença, na Biblioteca Nazionale Centrale, Landau Finaly Planisfero Rosselli [http://www.bncf.firenze.sbn.it/notizie/Cartografia\%20Web/ Rinascimento/Rosselli/RossellimA.htm]. Para uma discussão e ilustração do mapa ver Roberto Almagià, "On the Cartographic Work of Francesco Rosselli," Imago Mundi 8 (1951), p. 27-34, esp. 31-32 [http://www.jstor.org/stable/1150046]; e Tony Campbell, The Earliest Printed Maps, 1472-1500 (London: British Library, 1987), pp. 70-78, esp. 72-74. O planisfério de Martellus-Rosselli é também discutido e ilustrado em Guglielmo Cavallo, ed., Cristoforo Colombo e l'apertura degli spazi: Mostra storico-cartografica (Rome: Istituto Poligrafico e Zecca dello Stato, 1992), vol. 1, pp. 521-524; e em Rodney W. Shirley, The Mapping of the World: Early Printed World Maps, 1472-1700 (London: Holland Press, 1983), \#18, pp. 16-17. A ilha de Madagascar tem também esta forma de "L" no globo de 1492 de Martin Behaim (Cf. o Objektkatalog der Sammlungen des Germanischen Nationalmuseums [http://objektkatalog.gnm.de/objekt/WI1826]), provavelmente por influência de Martellus. 
12. A edição de 1513 da Geografia de Ptolomeu foi publicada por Johann Schott e é titulada Claudii Ptolemei viri Alexandrini Mathematic[a]e discipline Philosophi doctissimi Geographi[a]e opus. Um PDF duma copia com mapas coloridos à mão pode descarregar-se da Digitale Sammlungen da Bayerische Staatsbibliothek [http://daten.digitale-sammlungen.de/bsb00008750/image_1], e imagens de alta resolução de todas as páginas dum outro exemplar do livro podem ser acessadas via o catálogo da Biblioteca do Congresso [http://www.loc.gov/item/48041351]. Para uma discussão do mapa do Almirante ver Samuel McCoskry Stanton, "The Admiral's Map: What Was It? And Who the Admiral?" Isis 22.2 (1935), pp. 511-515 [http://www.jstor.org/stable/225132].

13. A carta de Caverio está em Paris, Biblioteca Nacional da França, Cartas e planos, SH archives 1 [http://gallica.bnf.fr/ark:/12148/btv1b7759102x]. A carta está bem reproduzida em Kenneth Nebenzahl, Atlas of Columbus and the Great Discoveries (Chicago: Rand McNally, 1990), pp. 41-43; e em escala maior no fac-similar preto-e-branco de onze folhas que acompanha o estudo do mapa de Edward L. Stevenson's, Marine World Chart of Nicolo de Caneiro Januensis 1502 (circa) (New York: American Geographical Society and the Hispanic Society of America, 1908).

14. Para uma discussão do uso da carta de Caverio na elaboração do mapa de 1507 de Waldseemüller ver Joseph Fischer e Franz Ritter von Wieser, Die älteste Karte mit dem Namen Amerika aus dem Jahre 1507 und die Carta marina aus dem Jahre 1516 des M. Waldseemüller (Ilacomilus) (Amsterdam: Theatrum orbis terrarum, 1968), pp. 26-29.

15. Para uma discussão acerca do globo de Schöner de 1515 ver Chet Van Duzer, Johann Schöner's Globe of 1515: Transcription and Study (Philadelphia: American Philosophical Society, 2010) = Transactions of American Philosophical Society 100.5.

16. Para uma análise do mapa Stevens-Brown ver Henry Newton Stevens, The First Delineation of the New World and the First Use of the Name America on a Printed Map (London: H. Stevens, Son and Stiles, 1928). Este livro está resenhado por exemplo em Geographical Journal 74.1 (1929), pp. 75-77. Uma discussão mais recente do mapa pode encontrar-se em Toby Lester, The Fourth Part of the World: The Race to the Ends of the Earth, and the Epic Story of the Map That Gave America its Name (New York: Free Press, 2009), pp. 399-403. A imagem pode ser acessada através da Coleção de Mapas da Biblioteca John Carter Brown [http://jcb.lunaimaging.com/luna/servlet/s/9nz9qy].

17. Ver Rodney W. Shirley, "Karte der Britischen Inseln von 1513: eine der ersten farbig gedruckten Karten," Cartographica Helvetica 19-20 (1999), pp. 13-17; e Jean-Marie Gérardin, "1508-2008: A propos de la première carte imprimée du duché de Lorraine et du Vastum Regnum," L'Annuaire de la Société du Val de Villé 33 (2008), pp. 57-77. A copia do Ptolomeu de 1513 da Divisão de Geografia e Mapas da Biblioteca do Congresso é uma das que tem o mapa impresso colorido das Ilhas Britânicas, mas infelizmente a imagem deste mapa disponível através do catálogo on-line da Biblioteca foi tomada preto e branco [http://memory.loc.gov/cgi-bin/ map_item.pl?data=/home/www/data/gmd//gmd3m/g3200m/g3200m/gct00262/

ca000079.jp2\&style=gmd\&itemLink=r?ammem/

gmd:@field\%28NUMBER+@band\%28g3200m+gct00262\%29\%29\&title=Claudii\%20Ptolemei\%20viri\%20Alexandrini\%20mathematice\%20di \%20ceteris\%20ante\%20lucubratorum\%20multo\%20prestantius\%20...+-

+Map\%20of\%20Great\%20Britain].

\section{RESUMOS}

Junho passado, Gerlinde Geiselmann e Elke Humml, bibliotecárias da Ludwig-MaximiliansUniversität (LMU) em Munique, descobriram um exemplar até agora desconhecido do mapa em 
gomos globulares (globe gores) desenhado originalmente pelo cartógrafo alemão Martin Waldseemüller (c. 1475-1520). O exemplar tinha sido encadernado no século XIX entre duas cópias da Perspectiva communis de Juan Peckham (uma publicada em 1504 e a outra por volta de 1510). Infelizmente, nada mais sabemos acerca da procedência deste exemplar do mapa em gomos. Até o momento conheciam-se unicamente outras quatro cópias destes gomos globulares, tratando-se portanto dum achado sensacional, tanto mais quanto este jogo de gomos é diferente dos outros. O propósito deste artigo é fornecer alguns detalhes acerca dos gomos e do significado da sua descoberta.

El pasado mes de junio, Gerlinde Geiselmann y Elke Humml, bibliotecarias de la Universidad Ludwig-Maximilians (LMU) en Múnich, descubrieron un ejemplar hasta ahora desconocido del mapa en gajos (globe gores) diseñado por el cartógrafo alemán Martin Waldseemüller (c. 1475-1520). El ejemplar había sido encuadernado en el siglo XIX entre dos copias de la Perspectiva communis de Juan Peckham (una publicada en 1504 y la otra alrededor de 1510). Desafortunadamente, no sabemos nada más acerca de la procedencia de este ejemplar del mapa en gajos. Hasta el momento sólo se conocían otros cuatro ejemplares de estos gajos globulares, lo que hace que se trate de un hallazgo sensacional, más aún considerando que este juego de gajos es diferente a los otros. El propósito de este artículo es comentar algunos detalles acerca de los gajos y del significado de su descubrimiento.

Dernière Juin, Gerlinde Geiselmann et Elke Humml, bibliothécaires de la Ludwig-MaximiliansUniversität (LMU) de Munich, ont découvert une copie jusque-là inconnue de la carte en quartiers globulaires (globe gores) conçue à l'origine par le cartographe allemand Martin Waldseemüller (c. 1475-1520). La copie avait été lié au XIXe siècle entre deux copies de la Perspectiva communis de Juan Peckham (un publié en 1504 et l'autre vers 1510). Malheureusement, nous ne savons rien de plus sur la provenance de cette exemplaire de la carte em quartiers. Jusqu'à présent, seulement quatre autres exemplaires de ces quartiers globulaires étaient connues: il s'agit d'une découverte sensationnelle, d'autant plus que ce jeu de quartiers est différent des autres. Le but de cet article est de fournir quelques détails sur la carte et l'importance de sa découverte.

This past June, Gerlinde Geiselmann and Elke Humml, librarians at Ludwig-MaximiliansUniversität (LMU) in Munich, discovered a previously unknown exemplar of globe gores originally designed by the german cartographer Martin Waldseemüller (c. 1475-1520). In the nineteenth century this set of the gores had been bound between two copies of Johannes Peckham's Perspectiva communis, one published in 1504, and the other in about 1510. No more is known about the provenance of the gores, unfortunately. Only four other exemplars of these globe gores were previously known to exist, so this was a sensational find-all the more so because this set of gores is different from the others. The purpose of the article is to give some details about the gores and the significance of this discovery. 
ÍNDICE

Palavras-chave: Martin Waldseemüller, gomos globulares, cartografia, Renascimento, século XVI, descoberta, Universidade Ludwig-Maximilians

Palabras claves: Martin Waldseemüller, gajos globulares, cartografía, Renacimiento, siglo XVI, descubrimiento, Universidad Ludwig-Maximilians

Índice cronológico: 1475, 1520

Índice geográfico: Mundo, Munique

Keywords: Martin Waldseemüller, globe gores, cartography, Renaissance, sixteenth century, discovery, Ludwig-Maximilians-Universität

Mots-clés: Martin Waldseemüller, quartiers globulaires, cartographie, Renaissance, XVIe siècle, découvertes, Ludwig-Maximilians-Universität

\section{AUTORES}

\section{CHET VAN DUZER}

Chet Van Duzer é pesquisador convidado da biblioteca John Carter Brown em Providence, Rhode Island. Tem escrito extensamente sobre mapas medievais e renascentistas em revistas como Imago Mundi, Terrae Incognitae y Word \& Image. Também é autor de Johann Schöner's Globe of 1515: Transcription and Study (2010), primeira análise detalhada de um dos mais antigos globos terrestres incluindo o Novo Mundo que sobrevivem. Boa parte de seu trabalho tem envolvido a procura das fontes tanto dos textos como das imagens de mapas antigos. Atualmente, é pesquisador residente no Centro John W. Kluge Center da Biblioteca do Congresso e trabalha no estudo detalhado da Carta marina de Waldseemüller [http://myloc.gov/Exhibitions/ EarlyAmericas/Interactives/Maps/html/cartamarina1516/index.html], assim como na finalização de um estudo sobre o grande mapa do mundo de Henricus Martellus de c. 1491 que se encontra em Yale [http://beinecke.library.yale.edu/dl_crosscollex/brbldl_getrec.asp? fld=img\&id=1040214]. Seu livro Sea Monsters on Medieval and Renaissance Maps está para ser publicado pela British Library em 2013.

chet.van.duzer@gmail.com 